\title{
The Isotope Effect of Disordered Superconductors
}

\author{
M. MieRzYŃSKA AND K.I. WYSOKIŃSKI \\ Institute of Physics, M. Curie-Skłodowska University \\ pl. M. Curie-Skłodowskiej 1, 20-031 Lublin, Poland
}

\begin{abstract}
The isotope effects in disordered weak and strong coupling superconductors of different symmetries of the order parameter have been studied. We have found that even though weak localisation corrections to Eliashberg equations describe a strong degradation of transition temperature with disorder of $s$-wave superconductors, their influence on isotope coefficient is relatively weak. On the other hand the calculated change of relative isotope coefficient with $T_{\mathrm{c}} / T_{\mathrm{c} 0}$ in homogeneous thin film materials is similar to that observed in high temperature superconductors.
\end{abstract}

PACS numbers: 74.25.Bt, 74.62.-c, 74.62.Dh

\section{Introduction}

The response of clean superconductors to intentionally introduced impurities provides the first information on the symmetry of the order parameter. In high temperature superconductors (HTS) [1] the isotope effect [2-4] shows complex behaviour: it takes on very small values for optimally doped samples and increases with decreasing oxygent content $\delta$ in $\mathrm{YBa}_{2} \mathrm{Cu}_{3} \mathrm{O}_{7-\delta}$ and decreasing transition temperature. Similar behaviour is observed in samples doped with such metals as Fe, Co, Zn, etc.

The complex behaviour is partially related to the layered structure of the materials and to the fact that some impurities go into the $\mathrm{CuO}$ conducting planes while others to the insulating planes. At the same time by changing the oxygen content one introduces disorder into the material. The very observation of the small but nonvanishing isotope coefficient is an indication of the lattice contribution to the mechanism of superconductivity.

It is the purpose of this paper to report on our recent studies of the isotope coefficient (IC) of impure superconductors with different symmetries of the order parameter. We shall comment on the role of in-plane and out-of-plane impurities [5] in changing $T_{\mathrm{c}}$ and IC of superconductors with $d$ - and $p$-wave symmmetries 
(Sec. 2). The weak localisation corrections and the effects of increased Coulomb repulsion in disordered bulk and thin film $s$-wave superconductors [6] are discussed in Sec. 3.

\section{In-plane and out-of-plane impurities in $d$ - and $p$-wave superconductors}

High temperature superconductors are believed to have a $d$-wave order parameter. The relative insensitivity of their superconducting transition temperature, to the dopant impurities, has caused a debate in the literature [7]. Eventually it has been proposed that both oxygen in 123 phase and $\mathrm{Sr}$ in $\mathrm{La}_{2-x} \mathrm{Sr}_{x} \mathrm{CuO}_{4}$ go mainly to the insulating $\mathrm{BaO}$, respectively, SrO layers and cause the forward scattering which has a little influence on the transition temperature. It is because the out-of-plane impurities are much less effective pair-breakers. The critical angle $\Theta_{c}$ around the forward scattering direction in which quasiparticles are scattered does depend on the Fermi wave vector and the distance of the impurities from the conduction plane. The first nonvanishing correction to the pair breaking parameter in $d$-wave supercondcutors has been found [8] to be of the third power of $\Theta_{c}$. Technically this means that the scattering time for out-of-plane impurities is reduced by this factor.

Recently we have calculated changes of $T_{\mathrm{c}}$ and IC due to out-of-plane impurities for different symmetry superconductors. The details of the calculations

TABLE

The changes of $T_{\mathrm{c}}$ and isotope coefficient $\alpha$ for different symmetries of the order parameter; $\rho_{\mathrm{c}}$ is the pair breaking parameter and $\Theta_{\mathrm{c}}$ - the critical angle for the forward scattering by the out-of-plane impurities.

\begin{tabular}{|c|c|c|}
\hline $\begin{array}{l}\text { Location of } \\
\text { impurities }\end{array}$ & $\begin{array}{c}d \text {-wave } \\
\Delta(\boldsymbol{k})=\Delta_{0} \cos (2 \phi)\end{array}$ & $\begin{array}{c}p \text {-wave } \\
\Delta(\boldsymbol{k})=\Delta_{0} \cos (\phi)\end{array}$ \\
\hline in-plane & $\begin{array}{c}\ln \left(T_{\mathrm{c}} / T_{\mathrm{c} 0}\right)=\psi\left(\frac{1}{2}\right)-\psi\left(\frac{1}{2}+\rho_{\mathrm{c}}\right) \\
\rho_{\mathrm{c}}=1 /\left(2 \pi \tau_{\mathrm{in}} k_{\mathrm{B}} T_{\mathrm{c}}\right) \\
\alpha_{0} / \alpha=1-\rho_{\mathrm{c}} \psi^{\prime}\left(\frac{1}{2}+\rho_{\mathrm{c}}\right)\end{array}$ & $\begin{array}{c}\ln \left(T_{\mathrm{c}} / T_{\mathrm{c} 0}\right)=\psi\left(\frac{1}{2}\right)-\psi\left(\frac{1}{2}+\rho_{\mathrm{c}}\right) \\
\rho_{\mathrm{c}}=1 /\left(2 \pi \tau_{\mathrm{in}} k_{\mathrm{B}} T_{\mathrm{c}}\right) \\
\alpha_{0} / \alpha=1-\rho_{\mathrm{c}} \psi^{\prime}\left(\frac{1}{2}+\rho_{\mathrm{c}}\right)\end{array}$ \\
\hline $\begin{array}{l}\text { out-of- } \\
\text {-plane }\end{array}$ & $\begin{array}{c}\ln \left(T_{\mathrm{c}} / T_{\mathrm{c} 0}\right) \\
=-\left(\Theta_{\mathrm{c}}^{3} / 6 \pi k_{\mathrm{B}} \tau_{\text {out }} T_{\mathrm{c}}\right) \psi^{\prime}\left(\frac{1}{2}\right) \\
\alpha_{0} / \alpha=1+\ln \left(T_{\mathrm{c}} / T_{\mathrm{c} 0}\right)\end{array}$ & $\begin{array}{c}\ln \left(T_{\mathrm{c}} / T_{\mathrm{c} 0}\right) \\
=-\left(\Theta_{\mathrm{c}}^{3} / 24 \pi k_{\mathrm{B}} \tau_{\mathrm{out}} T_{\mathrm{c}}\right) \psi^{\prime}\left(\frac{1}{2}\right) \\
\alpha_{0} / \alpha=1+\ln \left(T_{\mathrm{c}} / T_{\mathrm{c} 0}\right)\end{array}$ \\
\hline $\begin{array}{l}\text { in- and } \\
\text { out-of- } \\
\text {-plane }\end{array}$ & $\begin{array}{c}\ln \left(T_{\mathrm{c}} / T_{\mathrm{c} 0}\right)=\psi\left(\frac{1}{2}\right)-\psi\left(\frac{1}{2}+\rho_{\mathrm{c}}\right) \\
\rho_{\mathrm{c}}=1 /\left(2 \pi \tau_{\text {in }} k_{\mathrm{B}} T_{\mathrm{c}}\right) \\
+\Theta_{\mathrm{c}}^{3} /\left(3 \pi \tau_{\text {out }} k_{\mathrm{B}} T_{\mathrm{c}}\right) \\
\alpha_{0} / \alpha=1-\rho_{\mathrm{c}} \psi^{\prime}\left(\frac{1}{2}+\rho_{\mathrm{c}}\right)\end{array}$ & $\begin{array}{c}\ln \left(T_{\mathrm{c}} / T_{\mathrm{c} 0}\right)=\psi\left(\frac{1}{2}\right)-\psi\left(\frac{1}{2}+\rho_{\mathrm{c}}\right) \\
\rho_{\mathrm{c}}=1 /\left(2 \pi \tau_{\text {in }} k_{\mathrm{B}} T_{\mathrm{c}}\right) \\
+\Theta_{\mathrm{c}}^{3} /\left(12 \pi \tau_{\text {out }} k_{\mathrm{B}} T_{\mathrm{c}}\right) \\
\alpha_{0} / \alpha=1-\rho_{\mathrm{c}} \psi^{\prime}\left(\frac{1}{2}+\rho_{\mathrm{c}}\right)\end{array}$ \\
\hline
\end{tabular}


have been presented elswhere [5]. In the Table we collected the results for the $T_{\mathrm{c}}$ degradation and IC changes of superconductors.

In spin singlet $d$-wave or spin triplet $p$-wave superconductors in-plane nonmagnetic impurities are effective pair breakers (as are magnetic impurities in $s$-wave superconductors) and they have a large effect on the isotope coefficient which increases with decreasing superconducting transition temperature.

It is an interesting observation that the effect of out-of-plane impurities on IC when calculated as a function of relative change of $T_{\mathrm{c}}$ follows virtually the same universal curve as found for in-plane pair breakers but is limited to a smaller parameter region. The details have been published elsewhere [5].

\section{Disordered $s$-wave strong coupling bulk and thin film superconductors}

The superconducting transition temperature of a number of classic $s$-wave superconductors has been found to diminish after the material is made disordered. Weak localisation (WL) phenomena, connected inter alia with increased return probability or decreased diffusivity were proposed to be responsible for this kind of behaviour. Theoretical calculations with a single fitting parameter quite successfully explained the dependence of $T_{\mathrm{c}}$ on the residual resistivity $\rho$. In a recent paper [6] we have studied the effect of WL on the isotope coefficient. It turns out that the weak localisation effects introduce modifications of the interaction parameters in a superconductor and thus affect the transition temperature and related characteristics of disordered material. The calculations [6] show that WL, as a rule, decreases IC of electron-phonon superconductor. Figure 1 shows a typical kind of behaviour.

The corrections to BCS transition temperature due to disorder in two-dimensional superconductors have been a subject of studies and a matter of some controverses. The correction to leading logarithmic orders has been found to read $[9]$

$$
\ln \frac{T_{\mathrm{c}}}{T_{\mathrm{c} 0}}=-R_{\mathrm{s}} A_{3} \ln ^{3}\left(T_{0} / T_{\mathrm{c} 0}\right)-R_{\mathrm{s}} A_{2} \ln ^{2}\left(T_{0} / T_{\mathrm{c} 0}\right)+\ldots,
$$

where $T_{0}$ is some microscopic temperature scale, $A_{2}$ is a non-universal constant and $R_{\mathrm{s}}$ is the sheet resistance measured in units of $12 \pi \hbar / e^{2}$. The coefficient $A_{3}$ was claimed to take on a universal numerical value +1 . The correct calculations of the fluctuation contribution in the presence of strong spin-orbit coupling lead to $A_{3}=0$ [9]. Without spin-orbit scattering $A_{3}$ is nonzero and assumes a non-universal value. The curves in Fig. 2 show the change of the IC with decreasing $T_{\mathrm{c}}$ for thin films with $A_{3}=0$ and a number of values of $\sqrt{A_{2} R_{\mathrm{s}}}=0.01,0.45,0.80,1.35,1.85$ (from the bottom curve). The experimental points refer to HTS. We do not claim that due to two-dimensional nature of the conducting planes HTS should be considered as thin films. It is merely to show 

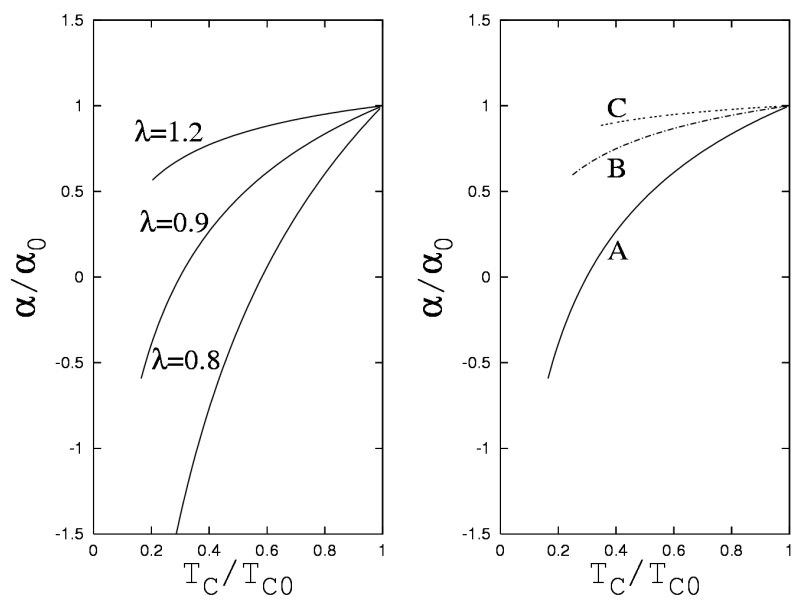

Fig. 1. Typical dependence of the relative change of IC $\alpha / \alpha_{0}$ vs. $T_{\mathrm{c}} / T_{\mathrm{c} 0}$ induced by weak localisation effects. For this calculation we have taken three values of the electron-phonon coupling $\lambda=0.8,0.9,1.2$, Coulomb pseudopotential $\mu=0.45$ and the ratio between Fermi energy $E_{\mathrm{F}}$ and Debye frequency $\omega_{\mathrm{D}}$ given by $\ln \left(E_{\mathrm{F}} / \omega_{\mathrm{D}}\right)=2$ (left panel); and $\lambda=0.9, \mu=0.45, \ln \left(E_{\mathrm{F}} / \omega_{\mathrm{D}}\right)=2(\mathrm{~A}), 3(\mathrm{~B}), 5(\mathrm{C})$ (right panel).

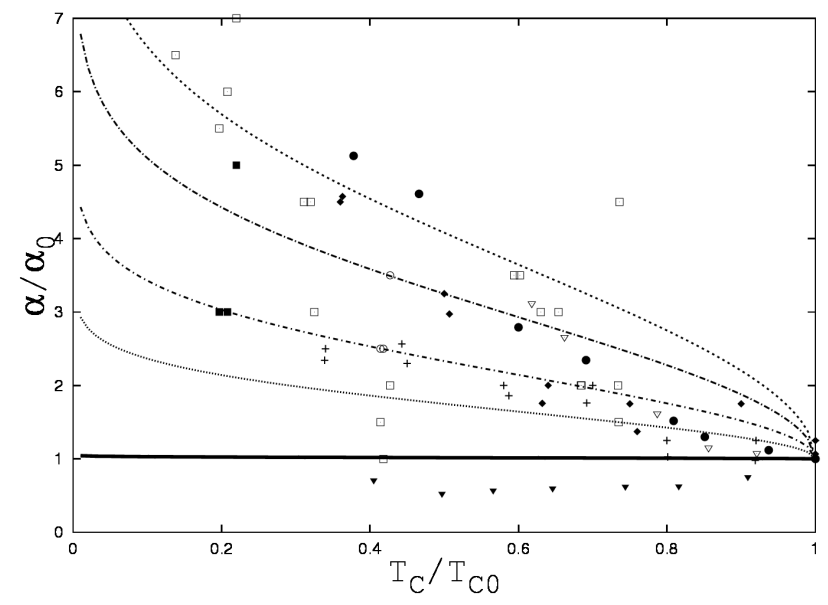

Fig. 2. The relative change of IC $\alpha / \alpha_{0}$ vs. $T_{\mathrm{c}} / T_{\mathrm{c} 0}$ in thin films characterised by the value of the parameters $\left(A_{3}=0\right) A_{2}$ and $R_{\mathrm{s}}$ such that $\sqrt{A_{2} R_{\mathrm{s}}}=0.01,0.45,0.80,1.35$, 1.85 (starting from the bottom curve). The points are experimental data on materials: optimally doped $\mathrm{La}_{1.85} \mathrm{Sr}_{0.15} \mathrm{Cu}_{1-x} \mathrm{M}_{x} \mathrm{O}_{4}$ with $\mathrm{M}=\mathrm{Co}$ (open triangles), Zn (filled diamonds) and $\mathrm{Ni}$ (crosses); overdoped $\mathrm{La}_{1.80} \mathrm{Sr}_{0.20} \mathrm{Cu}_{1-x} \mathrm{M}_{x} \mathrm{O}_{4}$ with $\mathrm{M}=\mathrm{Ni}$ (filled triangles), Fe (filled circles) [3]; $\mathrm{Y}_{1-x-y} \mathrm{Pr}_{x} \mathrm{Ca}_{y} \mathrm{Ba}_{2} \mathrm{Cu}_{3} \mathrm{O}_{7-\delta}$ with $x=0.2$ and $y=0.15$ (open circles), $y=0.25$ (filled squares); $\mathrm{YBa}_{2}\left(\mathrm{Cu}_{1-z} \mathrm{Zn}_{z}\right)_{3} \mathrm{O}_{7-\delta}$ (open squares) [4]. 


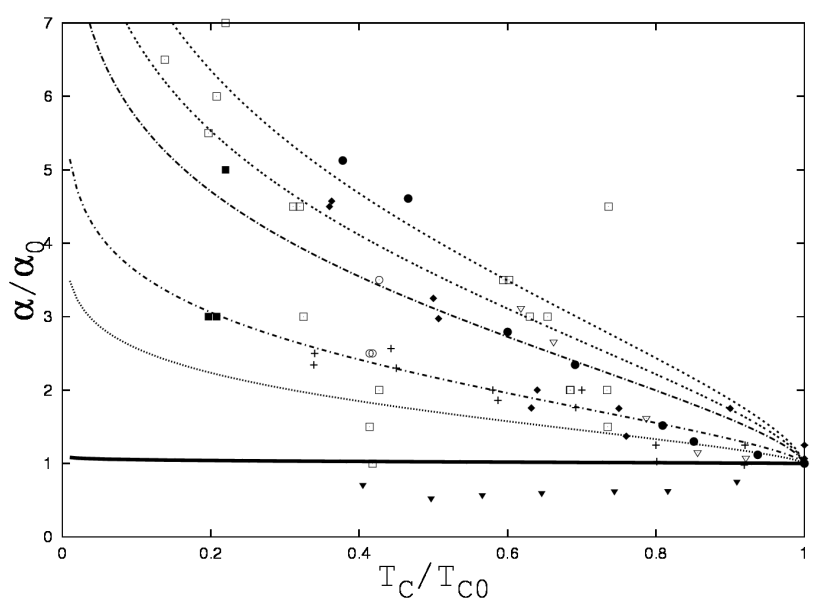

Fig. 3. $\alpha / \alpha_{0}$ vs. $T_{\mathrm{c}} / T_{\mathrm{c} 0}$ in thin films characterised by the value of the parameters $\left(A_{2}=0\right) A_{3}$ and $R_{\mathrm{s}}$ such that $\sqrt[3]{A_{3} R_{s}}=0.01,0.30,0.50,0.90,1.10,1.30$ (starting from the bottom curve). Experimental points are the same as in Fig. 2.

that two-dimensional aspects may be still important in these materials even in the superconducting state. A strong anisotropy of the superconducting state as measured by the ratio of the in-plane superconducting coherence length, $\xi_{a b} \approx 30 \AA$, to the coherence length in $c$-axis direction, $\xi_{\mathrm{c}} \approx 3 \AA$, may serve as a justification for the comparison with experiments.

Note that an equally good fit can be obtained for $A_{2}=0$ and $A_{3} \neq 0$ albeit for different set of parameters $A_{3} R_{\mathrm{S}}$ (see Fig. 3). Another objection against the comparison in Figs. 2 and 3 is the symmetry of the superconducting state. The calculations presented in this section all refer to $s$-wave superconductors and thus may not be appropriate for HTS believed to be $d$-wave symmetry. On the other hand there is an ongoing discussion on the symmetry of the order parameter. We think this result does not support neither of the symmetries. It merely says that to account for $T_{\mathrm{c}}$ degradation by disorder, various contributions have to be considered and they may induce big changes of IC.

\section{Summary and conclusions}

The natural question is to ask why the isotope effect of high temperature superconductors shows so complicated behaviour. In the present paper a number of corrections to the superconducting transition temperature induced by disorder have been discussed and the change of isotope coefficient calculated. We have found that impurity induced pair breaking in $d$-wave superconductors well accounts for the general dependence of $\alpha / \alpha_{0}$ vs. $T_{\mathrm{c}} / T_{\mathrm{c} 0}$ observed in high temperature superconductors. To the order the calculations are performed, the dependence is universal. The change of $T_{\mathrm{c}}$ does not depend on the location of the impurities and 
their "effectiveness to break the pairs" and as a result does not explain the scatter of the experimental points between different superconductors or different samples. This can be easily accounted for by the renormalisation group calculations exact to leading logarithmic order which introduce an additional parameter into the theory - the sheet resistance. Such calculations have been performed for thin film $s$-wave superconductors. Nevertheless the experimental data on bulk $d$-wave HTS can be easily reproduced with help of a single fitting parameter which is proportional to the sheet resistance of the material and is subject to change from sample to sample.

We have found that the weak localisation effects which lead to the degradation of $T_{\mathrm{c}}$ usually decrease IC below its canonical value given by the Eliashberg theory.

\section{Acknowledgments}

This work has been partially supported by the State Committee for Scientific Research (Poland) grant no. 2P03B 06225.

\section{References}

[1] J.G. Bednorz, K.A. Müller, Z. Phys. B 64, 189 (1986), Rev. Mod. Phys. 60, 585 (1988).

[2] K. Conder, Mater. Sci. Eng. R 32, 41 (2001).

[3] N.A. Babushkina, A.P. Zhernov, A.V. Inyushkin, T.N. Kulagina, V.I. Ozhogin, L.V. Molchanova, Physica C 272, 257 (1996).

[4] G. Soerensen, S. Gygax, Phys. Rev. B 51, 11848 (1995).

[5] M. Mierzyńska, K.I. Wysokiński, Phys. Rev. B 68, 1, 14504 (2003).

[6] M. Mierzyńska, K.I. Wysokiński, Study of the Isotope Effect of Disordered Strongly Coupled Superconductors, to be published.

[7] H. Eisaki, N. Kaneko, D.L. Feng, A. Damascelli, P.K. Mang, K.M. Shen, Z.-X. Shen, M. Greven, Phys. Rev. B 69, 064512 (2004).

[8] S. Chakavarty, H.-Y. Kee, E. Abrahams, Phys. Rev. B 67, 100504(R) (2003).

[9] S. Maekawa, H. Fukuyama, J. Phys. Soc. Jpn. 51, 1380 (1982); D. Belitz, T.R. Kirkpatrick, private communication. 